\title{
Prognosis and recurrence pattern of patients with cervical carcinoma and pelvic lymph node metastasis
}

\author{
Eliza Shrestha ${ }^{1}$, Xiong Ying ${ }^{1,2}$, Liang Li-Zhii, ${ }^{1,2}$, Zheng Min ${ }^{1,2}$ \\ Wang Luan-Hong ${ }^{1,2}$, Deng Peng-Fei ${ }^{1,2}$, Liu Ji-Hong ${ }^{1,2}$ * \\ 'State Key Laboratory of oncology in Southern China ${ }^{2}$ Department of Gynecologic Oncology, Cancer \\ Center, Sun Yat-sen University, Guangzhou, 510060, China
}

\begin{abstract}
Aim: To investigate the prognostic risk factor(s) and pattern of disease relapse of patients with cervical carcinoma and pelvic node metastasis.

Methods: One hundred twenty four cases of FIGOIBI IIA cervical carcinoma with pelvic node metastasis treated from January 1991 to December 2001 were selected for this study. Prognosis and recurrence were retrospectively analyzed using the clinico-pathological data.

Results: The overall 5 year survival and disease-free survival (DFS) was $63.3 \%$ and $61.4 \%$ respectively. Overall recurrence rate was 39.5\% (49/124). Intra-pelvic relapse $(25 / 41,61.0 \%)$ was significantly more frequent than extra-pelvic relapse $(13 / 41,1.7 \%, P=0.008)$. Multivariate analysis identified involvement of common iliac node as independent prognostic factor $(P=0.035)$. According to this factor, node-positive patients could be divided into low risk group (without common iliac node involvement, 104 cases) and high risk group (with common iliac node involvement, 20 cases). The DFS were $69.4 \%$ and $24.5 \%$ respectively, and the difference was significant $(P=0.003)$. Intra-pelvic relapse was observed in $22.1 \%$ of low risk and $25.0 \%$ of high risk group respectively, the difference was not significant $(P>0.05)$, however extra-pelvic relapse was seen in $7.7 \%$ of low risk and $40.0 \%$ of high risk group, and the difference was significant $(P<0.001)$.

Conclusions: Common iliac node involvement is the significant factor that influences the prognosis of patients with cervical carcinoma and pelvic node metastasis. According to this factor, survival and recurrence pattern differs significantly. These findings provide important reference for individualized modification and investigation of treatment mode.
\end{abstract}

Keywords: Cervical neoplasms; lymph node metastasis; prognosis

\section{Introduction}

In cervical cancer, pelvic lymph node metastasis is the universally accepted most important prognostic factor. About $40 \%$ of the patients who have positive pelvic lymph node(s) confirmed by radical surgery will eventually have tumor recurrence and 5-year survival range from $30 \%$ to $60 \%{ }^{1,2}$ How to improve the outcome of these patients is one the most important issues faced by gynecologic oncologists today. In this study, the clinical and pathological data of 124 cases of FIGO IB1-IIA cervical carcinoma with positive pelvic lymph node(s) were analyzed retrospectively to provide reference for the modification of individualized treatment by investigating factors that affect prognosis and recurrence pattern.

\section{Method}

\section{Case inclusion criteria}

The inclusion criteria of this study were as follows: 1) pathologically confirmed cervical cancer, no past or present history of other malignancies; 2) initially treated in our center, whose complete and analyzable clinical records are available; 3 ) a follow-up period of not less

Correspondence

Dr Eliza Shrestha enrolled as PhD in Gynecologic Oncology

Sun Yat Sen Medical University, 74 Zhong Shan,Road 2 Guangzhou, PR China 510080

E-mail: elizashrestha@hotmail.com, Cell: +86 13076772716 
than 60 months, with clear follow-up results (such as no evidence of disease, recurrence or death). After reviewing clinical records of patients admitted between Jan 1991 and Dec 2001 in the clinical data base of Dept. Gynecologic Oncology, cancer center of Sun Yat Sen University, a total of 124 cases of patients who met the above criteria was enrolled in this study.

\section{Treatment}

Fifty six patients with bulky tumor who were not suitable for primary surgical resection were given preoperative brachytherapy, with a mean dose of 16Gy (6-30Gy). Surgery was performed 2 to 3 weeks after radiotherapy.

All the patients underwent Piver II hysterectomy plus bilateral pelvic lymphadenectomy. The points of the surgery: separate the Douglas fossa and bilateral pararectal fossa, clamp and cut $2-3 \mathrm{~cm}$ of the bilateral uterosacral ligament. Paravesical fossa is not developed. Severe and ligate the uterine artery at the point where it crosses the ureter, thus open the superficial layer of the uterovesical ligament leaving the deeper layer intact. Pushing the terminal part of the ureter aside while pulling the uterus cephalad, clamp and cut the inner one third of the cardinal ligament. Cut the upper $1 / 3$ part of the vagina. Remove the adipolymphatic tissue surrounding the vessels from $2 \mathrm{~cm}$ above the bifurcation of the internal and external iliac artery to the deep iliac circumflex vein; the removed lymph nodes were grouped as common iliac, external iliac, deep inguinal, internal iliac and obturator node(s) according to anatomical location. The outer border of lymphadenectomy is the inner side of greater psoas muscle; the inner border is the ureter; the bottom is the surface of obturator nerve.

All patients received external radiation to the affected pelvic side wall postoperatively, and for those with deep stromal invasion of cervix or cervical canal, external radiation to the bladder field was added. The dose of radiation was 46-50Gy. Thirty six patients also received chemotherapy before and after the radiotherapy. Therefore in this study, 88 patients received adjuvant radiotherapy only and 36 patients received sequential chemotherapy and radiotherapy postoperatively. Thirty of the 36 patients received combined chemotherapy based on DDP or carboplatin; 6 cases received regimen containing 5-FuyBleomycin or CTX. The median cycles are 2 (1-4 cycles).

\section{Follow-up}

Patients were followed up every 1 to 3 months in the first year after treatment, every six months in the second year, and then every 12 months form the third year on. Follow up procedure included: gynecologic examination, rectal examination, imaging (Ultrasound or CT scan) and test for serum tumor markers such as squamous cell carcinoma antigen (SCCAg). Recurrence of disease was diagnosed according to the clinical symptoms and signs, imaging examination results and/ or pathology diagnosis, and the results of serological examination.

\section{Statistical methods}

SPSS10.0 software was used for statistical analysis. Chi -square test was used for comparison of emuerative data and life-table method for caculating 5-year disease-free survival (DFS). Kaplan-Meier method was used for drawing the survival curve and the log-rank for the test. Multivariate analysis using Cox regression model was used for survival analysis. A P value of $<0.05$ was considered to be a significant difference.

\section{Results}

\section{Clinical features}

According to the laterality of lymph node metastasis, anatomical location and the number of groups of positive node(s): unilateral metastasis was found in 78 patients, bilateral metastasis in 46 patients; common iliac lymph node metastasis in 20 patients, and non-common iliac lymph node metastasis in 104 patients; less than 3 groups of lymph node metastasis in 102 patients, $>3$ group lymph node metastasis in 22 patients. The distribution of other clinicopathological factors, such as age, FIGO stage is listed in (Table1).

\section{Prognosis of univariate and multivariate analysis}

The overall 5-year survival rate was $63.3 \%$; the 5-year disease-free survival rate was $61.4 \%$. As shown in Table 1 , in univariate analysis 5 -year disease-free survival were significantly correlated with groups of positive nodes (1-2 group, $\geq 3$ group), common iliac lymph node involvement $(P<0.05, P<0.05)$. Age, FIGO stage, histological type, gross type, tumor size, histological grading, deep myometrial invasion, preoperative adjuvant radiation, postoperative adjuvant treatment had no significant correlation with 5-year disease-free survival $(P>0.05)$. multivariate analysis using the Cox proportional hazard model showed that the common iliac lymph node involvement was the independent prognostic factor $(P=0.035)$. 
Table 1. Univariate analysis of prognosis

\begin{tabular}{|c|c|c|c|c|}
\hline \multicolumn{2}{|c|}{ Factors } & \multirow{2}{*}{$\begin{array}{c}\begin{array}{c}\text { Number } \\
\text { of case }\end{array} \\
25\end{array}$} & \multirow{2}{*}{$\begin{array}{c}\begin{array}{c}\text { Five Year Disease- } \\
\text { free Survival }(\%)\end{array} \\
53.7\end{array}$} & \multirow{2}{*}{$\begin{array}{c}P \\
0.221\end{array}$} \\
\hline $\begin{array}{l}\text { Age } \\
\leq 35\end{array}$ & $\begin{array}{l}\leq 35 \\
99\end{array}$ & & & \\
\hline FIGO stage & $\begin{array}{l}\text { IB1 } \\
\text { IB2 }\end{array}$ & $\begin{array}{l}56 \\
36\end{array}$ & $\begin{array}{l}66.6 \\
55.9\end{array}$ & 0.516 \\
\hline & IIA & 32 & 56.4 & \\
\hline Pathology & $\begin{array}{l}\text { Squamous cell carcinoma } \\
\text { Adenocarcinoma }\end{array}$ & $\begin{array}{l}98 \\
19\end{array}$ & $\begin{array}{l}65.4 \\
40.8\end{array}$ & 0.386 \\
\hline & Adeno Squamous & 7 & 71.4 & \\
\hline Gross type & $\begin{array}{l}\text { Exophytic } \\
\text { Endophytic }\end{array}$ & $\begin{array}{l}80 \\
44\end{array}$ & $\begin{array}{r}63.3 \\
58\end{array}$ & 0.522 \\
\hline Tumor size & $\begin{array}{l}\text { d" } 4 \mathrm{~cm} \\
\text { e" } 4 \mathrm{~cm}\end{array}$ & $\begin{array}{l}88 \\
36\end{array}$ & $\begin{array}{l}63.7 \\
53.6\end{array}$ & 0.486 \\
\hline Grading & $\begin{array}{l}\text { I - II } \\
\text { III }\end{array}$ & $\begin{array}{l}62 \\
62\end{array}$ & $\begin{array}{l}53.5 \\
70.6\end{array}$ & 0.238 \\
\hline Deep stromal invasion & $\begin{array}{l}\text { No } \\
\text { Yes }\end{array}$ & $\begin{array}{l}49 \\
75\end{array}$ & $\begin{array}{r}61.8 \\
61\end{array}$ & 0.969 \\
\hline Parametrial margin & $\begin{array}{l}\text { Negative } \\
\text { Positive }\end{array}$ & $\begin{array}{r}114 \\
10\end{array}$ & $\begin{array}{l}63.7 \\
24.7\end{array}$ & 0.255 \\
\hline Laterality of node metastasis & $\begin{array}{l}\text { Unilateral } \\
\text { Bilateral }\end{array}$ & $\begin{array}{l}78 \\
46\end{array}$ & $\begin{array}{l}68.9 \\
47.4\end{array}$ & 0.112 \\
\hline Common illiac node involvement & $\begin{array}{l}\text { No } \\
\text { Yes }\end{array}$ & $\begin{array}{r}104 \\
20\end{array}$ & $\begin{array}{l}69.4 \\
24.5\end{array}$ & 0.003 \\
\hline Group of lymph node metastasis & $\begin{array}{l}1-2 \\
\text { e"3 }\end{array}$ & $\begin{array}{r}102 \\
22\end{array}$ & $\begin{array}{l}66.5 \\
38.6\end{array}$ & 0.013 \\
\hline Postoperative adjuvant therapy & $\begin{array}{l}\text { Radiation } \\
\text { Radiation }+ \text { Chemotherapy }\end{array}$ & $\begin{array}{l}88 \\
36\end{array}$ & $\begin{array}{l}62.7 \\
58.5\end{array}$ & 0.232 \\
\hline Preoperative brachytherapy & $\begin{array}{l}\text { No } \\
\text { Yes }\end{array}$ & $\begin{array}{l}68 \\
56\end{array}$ & $\begin{array}{l}60.4 \\
63.1\end{array}$ & 0.608 \\
\hline
\end{tabular}

\section{Analysis of recurrence}

The median follow-up time for the patient was 70 months (48-120 months), no patient was lost in followup in this study. During follow-up, a total of 49 cases of recurrence were observed, the recurrence rate was $39.5 \%$ (49/124). Among which, 85.7\% (42/49) recurrence were observed within two years after the treatment. According to follow-up information, the site of tumor recurrence was clear in 41 cases, in which intra-pelvic relapse were 25 cases, accounting for $61.0 \%$ (25/41); extra pelvic relapse (including para-aortic lymph nodes) were 13 cases, accounting for $31.7 \%$ (13/41); and both intra and extra-pelvic relapse in 3 cases, accounting for $7.3 \%$ (3/41). Common sites of extra-pelvic recurrence included: left supraclavian lymph node ( 5 cases), lung (4 cases), liver ( 2 cases), bone ( 2 cases), Para-aortic lymph nodes (1 case), vulva (1case), and inguinal lymph nodes (1 case). Eight cases were via telephone and (or) follow-up letter confirmed death due to cancer, but the site of tumor recurrence was unknown. There were 42 cancer-related deaths in this group patient at the end of follow up. On statistical test, intra-pelvic recurrence were significantly greater than extra-pelvic recurrences $(25 / 41,13 / 41, \mathrm{P}=0.008)$.

\section{Prognostic risk grouping and the relationship between recurrent patterns}

Based on the results of multivariate analysis, 124 cases of pelvic lymph node-positive patients were divided into two groups according to the prognostic risk level. The high-risk group included 20 cases, characterized by common iliac lymph node involvement; the 5-year disease-free survival rate was $24.5 \%$. The low-risk group included 104 cases, characterized by non-common iliac node involvement, the five-year disease-free survival was $69.4 \%$, the difference was significant $(P=0.003)$.

The recurrence pattern of high and low risk group was shown in Table 2: intra-pelvic recurrence rate was $22.1 \%$ 
Table 2. Recurrence pattern by prognostic risk grouping

\begin{tabular}{cccccc}
\hline & & \multicolumn{4}{c}{ Recurrence } \\
\cline { 3 - 6 } Group & No of Cases & Intra-pelvic & Intra/extra-pelvic & Extra-pelvic & Unknown \\
\hline High Risk Group & 104 & 22 & 1 & 7 & 7 \\
Low Risk Group & 20 & 3 & 2 & 6 & 1 \\
\hline
\end{tabular}

$[(22+1) / 104]$ in low-risk group; while $25.0 \%[(3+2) /$ $20]$ in the high-risk group, the difference was not significant $(P>0.05)$. Extra-pelvic recurrence was $7.7 \%$ $[(7+1) / 104]$ in low-risk group; while $40.0 \%[(6+2) / 20]$ in the high-risk group, the difference was significant $(P<0.001)$.

\section{Discussion}

Among cervical cancer patients treated with radical surgery, those who had pelvic lymph node metastasis will undoubtedly have the worst prognosis. According to the report of our center, the 5 year disease free survival rate of node-positive patients was around $60.0 \%{ }^{3}$ However previous reports showed that even in node-positive patients, the prognosis still differs significantly according to some other factors such as the number or location of positive node(s). ${ }^{4-6}$ In this study, multivariate analysis demonstrated common iliac node involvement as independent prognostic factor, while groups of positive node(s) was significantly correlated with survival however failed in multivariate analysis. Therefore we use common iliac lymph node involvement for risk grouping of our node-positive patients in order to more accurately recognize high risk patient and make appropriate treatment plan to improve overall survival.

The overall recurrence rate in this study is $39.5 \%$, which is similar to previous report. ${ }^{1}$ As intra-pelvic recurrence is significantly greater than extra-pelvic recurrence, the insufficient local control of tumor is the main reason for treatment failure; this is especially obvious in lowrisk group. High risk group has a similar intra-pelvic recurrence rate but a significantly higher extra-pelvic recurrence rate compared with low risk group, which suggests the importance of awareness of occult distant metastasis and proper prevention of extra-pelvic failure particularly for high risk patients.

From the above results, it is not difficult to see that the key to improve the disease-free survival of nodepositive patients is through better local control of tumor. Experience accumulated from long-term clinical practice has proved cervical cancer is a kind of local-regional tumor; its early spread is through regional (pelvic) lymph node, while distant metastasis develops only in advanced stage. Therefore for clinical early-staged (FIGO IB1-IIA) cases, local treatment, such as surgery and/or radiotherapy can achieve satisfactory tumor control. Strategies to improve local control should as well be focused on surgery and radiation. In this study, all patients were treated with Piver II operation. Although there is one prospective randomized trial states Piver II and Piver III surgery can achieve similar long-term survival in FIGO IB-IIA patients, the question whether Piver II operation is a sufficient dissection for node-positive patients or not remains unanswered. Some authors have used the "giant section" technique to investigate the relationship between parametrial metastasis and pelvic node metastasis. They found three patterns of parametrial metastasis: direct invasion, vascular space involvement and metastasis to parametrial nodes, the latter two were the predominant patterns. Parametrial nodes distribute in cardinal ligament (52\%-63\%), uterovesical ligament (14\%-35\%) and uterosacral ligament $(9 \%)$, its metastasis could reach $30 \%$; all pelvic node-positive patients had parametrial metastasis. ${ }^{8}$ In another retrospective study (594 cases)[9], 47.9\% of pelvic node-positive patients had positive parametria, while only $6.0 \%$ of nodenegative patients had positive parametria. ${ }^{9}$ Parametrial metastasis had significant impact on recurrence, disease-free survival and overall survival. Puente $\mathrm{R}^{10}$ reported $36.4 \%$ and $9.4 \%$ parametrial metastasis rate in node-positive and node-negative patients respectively. $\mathrm{Li} \mathrm{B}^{11}$ have detected parametrial node in $63 \%(38 / 60)$ of cervical cancer patients using methylene blue, its distribution was similar to previous report, the mean diameter of parametrial node was less than $1 \mathrm{~cm}$, however $20 \%$ of them harbored metastasis. ${ }^{8}$ The above evidence suggests that parametrium is one of the most common sites of tumor metastasis and it is constantly correlated with pelvic lymph node metastasis. This rule can be used to individualize the extent of surgical resection: for example, the surgeon can take out the suspected pelvic node(s) for frozen section during operation, once metastasis is confirmed, the parametria should be excised along the pelvic side wall as recommended by Piver III dissection; otherwise a Piver II dissection can be considered to shorten the operation time and reduce surgery-related complications while risk little to leave behind positive parametria. If frozen section is unavailable, appropriate procedure should 
be taken after evaluating the risk of nodal metastasis according FIGO Stage, tumor size and serum SCC level. ${ }^{12,}{ }^{13}$ Since $80 \%$ of the metastatic nodes are less than $1 \mathrm{~cm}$ in diameter, imaging examination and intraoperative palpation is lack of sufficient sensitivity and therefore should not be used as proof for decision making.

Regarding radiotherapy, although all the patients in this study received external beam radiation, the radiation field was not unified. It was a kind of "patching" type of radiation compared with whole pelvis radiation. This is due to the different interpretation of pelvic node metastasis: does nodal metastasis in a certain anatomical site mean that site is the only place which contains sub-clinical residue that requires radiation? Or does it mean the tumor has spread beyond the original site (cervix) and the regional lymphatic system may harbor sub-clinical lesions? From the correlation between parametrial metastasis and pelvic node metastasis, it can be easily deduced that pelvic node metastasis is a histological parameter which indicates possible sub-clinical spread in pelvis and should not be interpreted as a sign of tumor residue in the corresponding site. In recent years, our center has switched to whole pelvis radiation to improve local control of node-positive patients. The effect remains to be seen. Other reports showed chemotherapy given concurrently with radiation was also helpful of improving long-term survival. ${ }^{14}$

We have reported that patients with pelvic lymph node metastasis usually develop distant metastasis after treatment, from this study; we further discovered that extra-pelvic recurrence was mainly seen in patients with common iliac node involvement. ${ }^{3}$ How to improve longterm survival of this subgroup of patients is an important problem. Since common iliac node metastasis often indicates nodal metastasis of next station of lymphatic drainage, it is necessary to consider taking out the para-aortic nodes once positive common iliac node is confirmed during operation, or giving preventive radiation postoperatively. Besides concurrent chemoradiation, whether adjuvant chemotherapy can improve survival or not deserves further investigation as well.

\section{Conclusion}

This study shows that patients with pelvic lymph node metastasis has poor prognosis. According to whether common iliac node is involved or not, the 5 year diseasefree survival and recurrence patterns differ significantly, these findings may provide important inspiration and reference for the modification and exploration of individualized treatment.

\section{References}

1. Fuller AF Jr, Elliott N, Kosloff C, et al. Determinants of increased risk for recurrence in patients undergoing radical hysterectomy for stage IB and IIA carcinoma of the cervix. Gynecol Oncol 1989; 33(1):34-9.

2. Monk BJ, Cha DS, Walker JL, et al. Extent of disease as an indication for pelvic radiation following radical hysterectomy and bilateral pelvic lymph node dissection in the treatment of stage IB and IIA cervical carcinoma. Gynecol Oncol 1994; 54 (1):4-9.

3. Xiong YÿLiang LZÿLiu JHÿet al. Stratification of prognostic factors for squamous cell carcinoma of the cervix and its clinical significance. Chinese Journal of Clinical Oncology 2007; 34(18): 10481052.

4. Aoki Y, Sasaki M, Watanabe M, et al. Highrisk group in node-positive patients with stage IB, IIA, and IIB cervical carcinoma after radical hysterectomy and postoperative pelvic irradiation. Gynecol Oncol 2000; 77(2): 305-9.

5. Morice $\mathrm{P}$, Castaigne D, Pautier P, et al. Interest of pelvic and paraaortic lymphadenectomy in patients with stage IB and II cervical carcinoma. Gynecol Oncol 1999; 73(1):106-10.

6. Lin HH,Cheng WF, Chan KW, et al. Risk factors for recurrence in patients with stage IB, IIA, and IIB cervical carcinoma after radical hysterectomy and postoperative pelvic irradiation. Obstet Gynecol 1996; 88(2):274-9.

7. Landoni F, Maneo A, Cormio G, et al. Class II versus class III radical hysterectomy in stage IBIIA cervical cancer: a prospective randomized study. Gynecol Oncol 2001; 80(1):3-12.

8. Benedetti-Panici P, Maneschi F, D'Andrea G, et al. Early cervical carcinoma: the natural history of lymph node involvement redefined on the basis of thorough parametrectomy and giant section study. Cancer 2000; 88(10):2267-74.

9. Wright JD, Grigsby PW, Brooks R, et al. Utility of parametrectomy for early stage cervical cancer treated with radical hysterectomy. Cancer 2007; 110(6):1281-6.

10. Puente R, Guzman S, Israel E, et al. Do the pelvic lymph nodes predict the parametrial status in cervical cancer stages IB-IIA? Int J Gynecol Cancer 2004, 14(5):832-40.

11. Li B, Wu LY, Li XG, et al. Lymphatic mapping and parametrial lymph nodes identification in patients with early stage cervical cancer. Zhonghua Fu Chan Ke Za Zhi 2006 Sep; 41(9):608-11.

12. Xiong Y, Liang LZ, ZhenM, et al. Correlation of serum squamous cell carcinoma antigen with 
clinico-pathological features and prognosis of squamous cell carcinoma of uterine cervix. Zhonghua Fu Chan Ke Za Zhi 2007 Jan; 42(1):2933.

13. Benedetti-Panici P, Maneschi F, Scambia G, et al. Lymphatic spread of cervical cancer: an anatomical and pathological study based on 225 radical hysterectomies with systematic pelvic and aortic lymphadenectomy. Gynecol Oncol 1996; 62(1):19-24.

14. Peters III WA, Liu PY, Barrett II RJ, et al. Concurrent chemotherapy and pelvic radiation therapy compared with pelvic radiation therapy alone as adjuvant therapy after radical surgery in highrisk early-stage cancer of the cervix. J Clin Oncol 2000; 18(8):1606-13. 